\title{
Subclinical Cushing's syndrome and clinical implications in bilateral compared to unilateral adrenal incidentalomas: a meta-analysis
}

\author{
Stavroula A. Paschou ${ }^{1}$, Eleni Kandaraki ${ }^{1}$, Fotini Dimitropoulou ${ }^{1}$, \\ Dimitrios G. Goulis ${ }^{2}$, Andromachi Vryonidou ${ }^{1}$ \\ ${ }^{1}$ Department of Endocrinology and Diabetes, Hellenic Red Cross Hospital, Athens, Greece \\ ${ }^{2}$ Unit of Reproductive Endocrinology, First Department of Obstetrics and Gynecology, Medical School, \\ Aristotle University of Thessaloniki, Thessaloniki, Greece
}

Introduction: The aim of this study was to systematically review the literature for studies that have investigated possible differences in prevalence of subclinical Cushing's syndrome (SCS) and related clinical implications between patients with unilateral (UAI) and bilateral adrenal incidentalomas (BAI) and to meta-analyze the best evidence available.

Methods: Electronic databases PubMed, MEDLINE and EMBASE were systematically searched. Main study outcome was the prevalence of SCS in patients with UAI and BAI. Secondary outcomes were the prevalence of obesity, diabetes, glucose intolerance, hypertension, dyslipidemia and osteoporosis in patients with UAI and BAI. Random effects odds ratios (OR) or standardized mean differences (SMD) and 95\% confidence intervals (CI) were calculated. Meta-analysis was conducted using Review Manager (RevMan 5.3).

Results:

- Six studies were included in the meta-analysis involving in total 1239 patients, 968 with UAI and 271 with BAI.
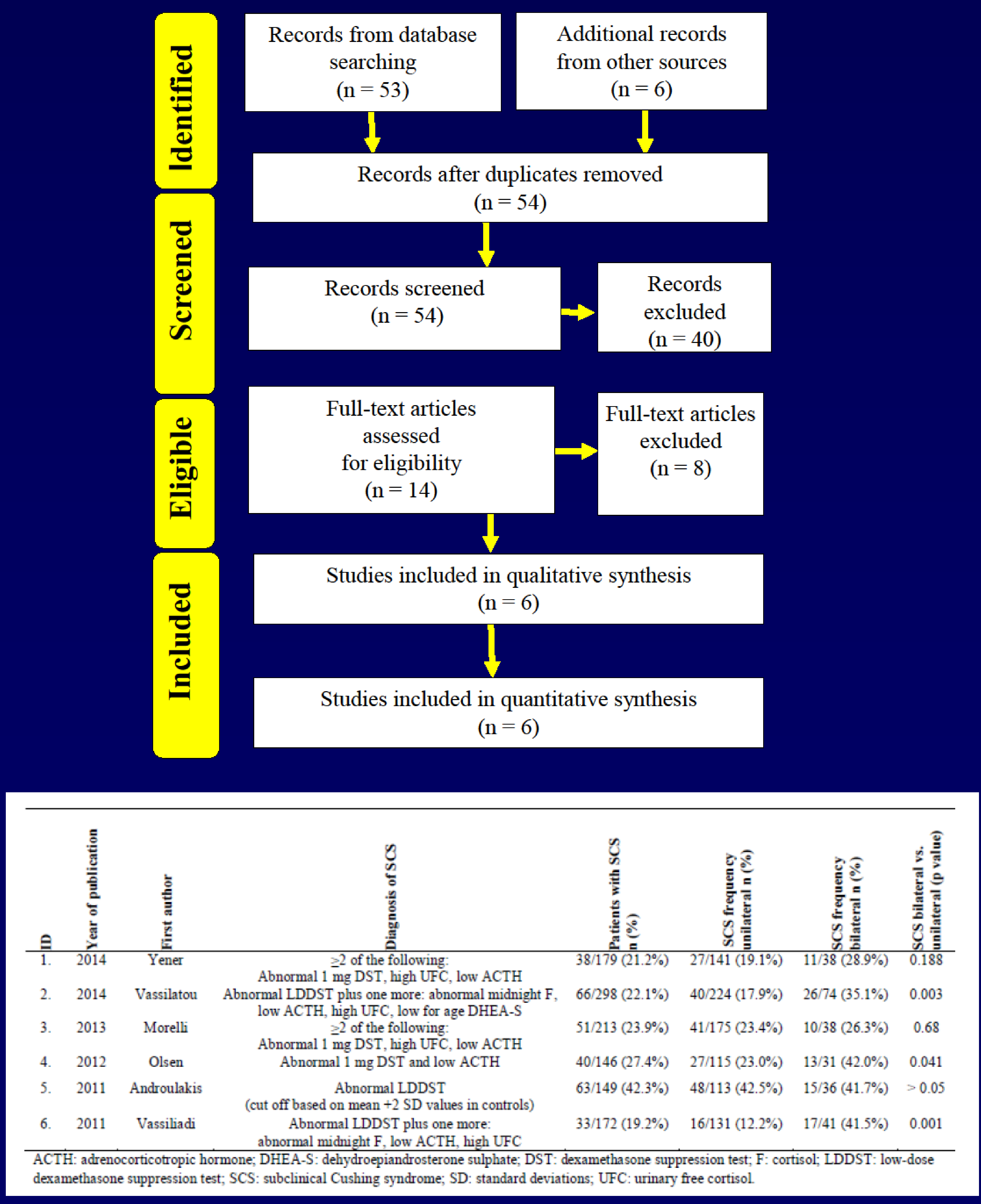

- Patients with UAI had lower prevalence of SCS compared with those with BAI [OR $(95 \% \mathrm{CI}) 0.51(0.32$; $\left.0.81), \mathrm{I}^{2}=55 \%\right]$.

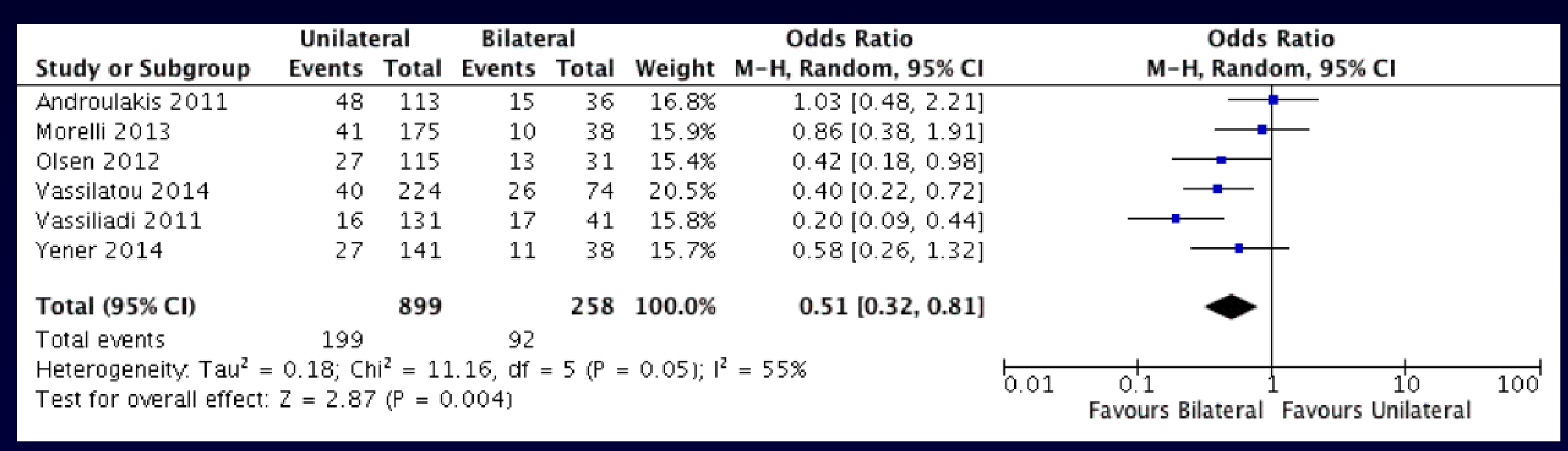

- The mass diameter of UAI did not differ from BAI (the size of the largest lesion) [SMD (95\% CI) $-0.40(-0.97$; $\left.0.17), \mathrm{I}^{2}=91 \%\right]$.

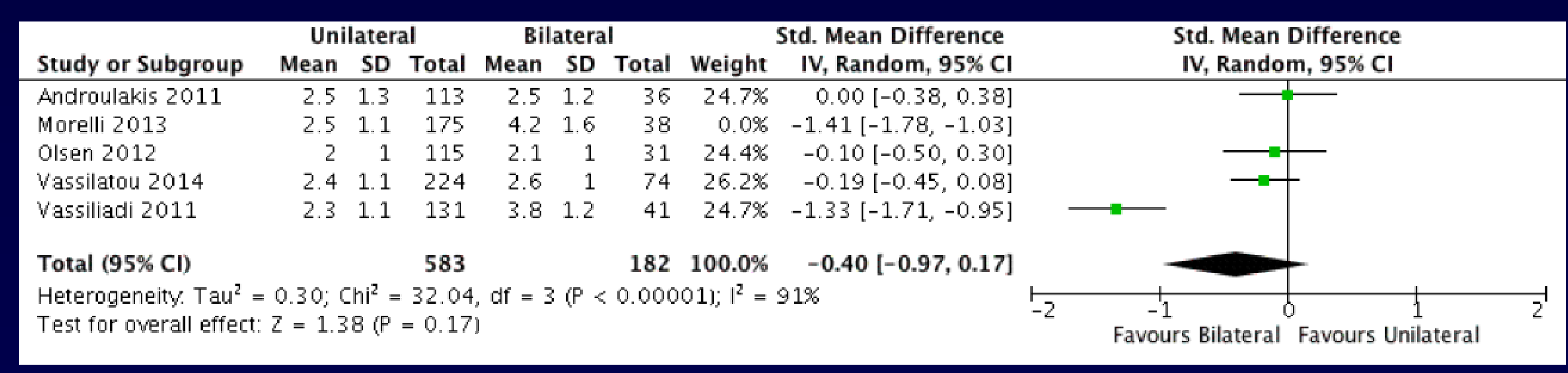

- The prevalence of obesity [SMD $(95 \% \mathrm{CI}) 0.06(-0.10$; $0.22), \mathrm{I}^{2}=0 \%$ ], diabetes [OR $(95 \% \mathrm{CI}) 0.78(0.51 ; 1.21)$, $\mathrm{I}^{2}=0 \%$ ], hypertension [OR $(95 \% \mathrm{CI}) 1(0.47 ; 2.12), \mathrm{I}^{2}=$ $74 \%$ ] and dyslipidemia [OR (95\% CI) $0.93(0.49 ; 1.78), \mathrm{I}^{2}$ $=49 \%$ ] did not differ between UAI and BAI.
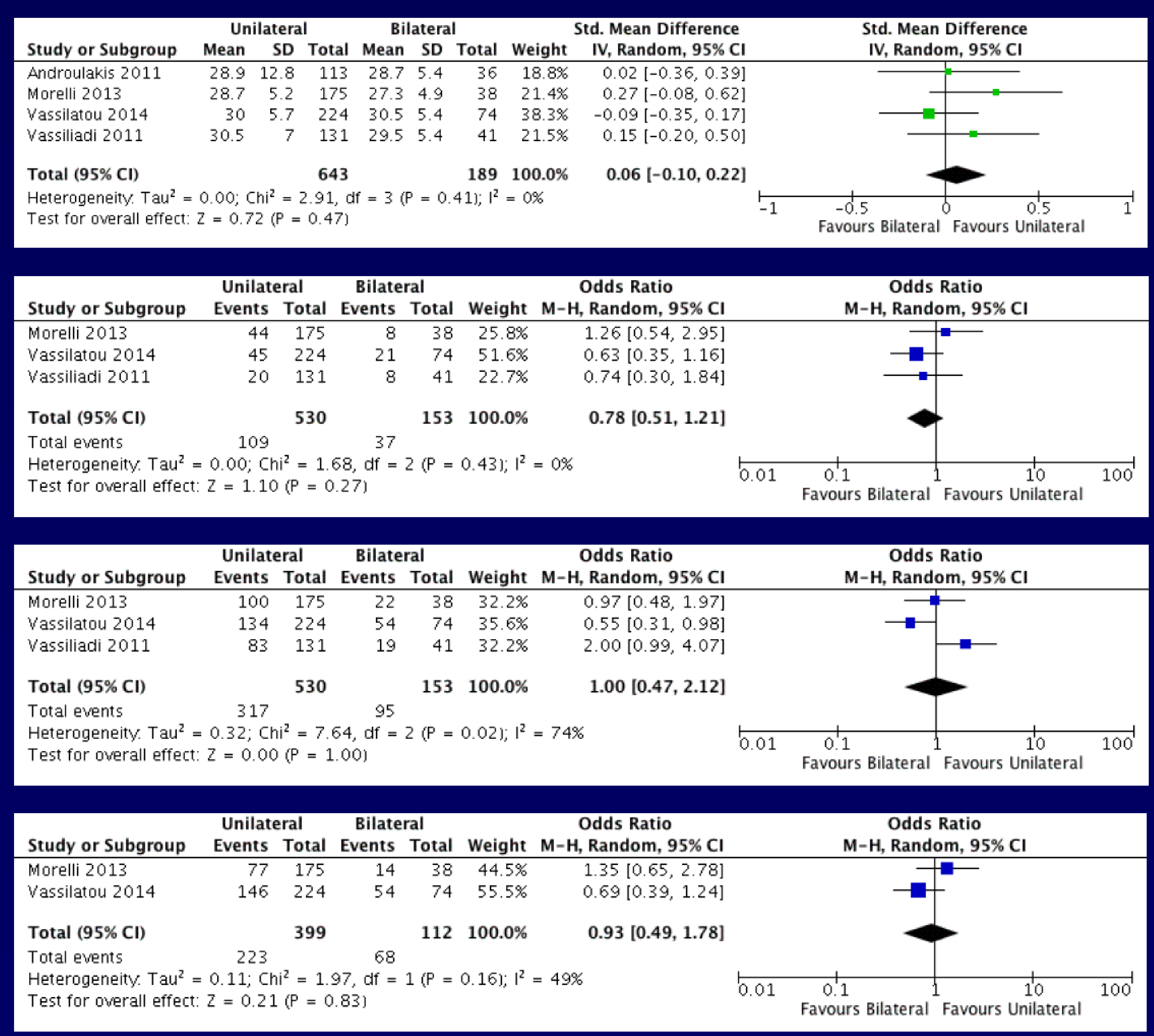

Conclusions: Patients with BAI present a higher prevalence of SCS compared to patients with UAI, without any differences in related clinical implications. 